\title{
A hybrid solid-liquid polymer photodiode for the bioenvironment
}

\author{
M. R. Antognazza, ${ }^{1}$ D. Ghezzi, ${ }^{1}$ D. Musitelli, ${ }^{2}$ M. Garbugli, ${ }^{2}$ and G. Lanzani ${ }^{2, a)}$ \\ ${ }^{1}$ Italian Institute of Technology, via Morego 30, 16163 Genova, Italy \\ ${ }^{2}$ Dipartimento di Fisica, Politecnico di Milano, P.zza L. da Vinci 32, 20133 Milano, Italy
}

(Received 22 October 2008; accepted 3 May 2009; published online 15 June 2009)

\begin{abstract}
We demonstrate that a prototypical semiconducting polymer, poly[2-methoxy-5(2'-ethylhexyloxy)-p-phenylene vinylene] (MEH-PPV) maintains unaltered its optoelectronic properties throughout the various steps for neural preparation. Films of MEH-PPV, after prolonged immersion in water or buffer solution, are characterized by linear and nonlinear optical spectroscopy. Based on this result, we introduce a hybrid solid-liquid photodiode based on MEH-PPV, in which we use culturing media as liquid, ionic cathodes. The hybrid device is proposed as an active interface between living tissue and conducting polymers for cell diagnostic and neural implants. () 2009 American Institute of Physics. [DOI: 10.1063/1.3153846]
\end{abstract}

In the past few decades, $\pi$-conjugated semiconducting polymers have been extensively studied, and a great variety of optoelectronic applications have been widely demonstrated. ${ }^{1}$ However, it is only recently that organic electronics has started to merge with biology and medicine, to (i) develop a new generation of biosensors, ${ }^{2}$ and (ii) to produce polymer-based coatings for neural interfaces and neuronstimulating electrodes. ${ }^{3}$ In contrast, very few examples of semiconducting polymers as active materials for neural prosthesis applications have been reported, ${ }^{4}$ despite the important potential for integration of polymer-based devices into living systems, for example organic photodiodes for artificial vision. ${ }^{5}$ Here, we introduce a hybrid solid-liquid photodiode, based on poly[2-methoxy-5-(2'-ethylhexyloxy)-p-phenylene vinylene] (MEH-PPV), as a candidate for direct interfacing with a neuronal network. The interaction between the conjugated polymer and a neural preparation, or in general a living tissue, requires the optoelectronic properties of polymers to remain unaltered during cell culture and, later, in the biological (or biological-like) environment.

In general, the presence of water represents a condition that cannot be disregarded. To this respect, the hydrophobic character of most semiconducting polymers might be seen as a substantial advantage. Nevertheless, it is actually well known that an effective encapsulation of organic devices is strictly required to protect them against moisture and oxygen, and to achieve adequate lifetimes. While the effect of oxygen has been reported in some cases, ${ }^{6}$ there are very few studies on the operation of semiconducting polymers which have been intentionally put in contact with water or, more generally, with some ionic solution. ${ }^{7}$

The main negative effects related to the contact with water/oxygen are as follows:

(i) the formation, inside the organic active layer, of nonemissive quenching species;

(ii) the degradation of the reactive, low work function metals used as cathodes.

Regarding to the first problem, we directly investigate the effect of immersion in water onto the photophysical proper-

${ }^{\text {a)} E l e c t r o n i c ~ m a i l: ~ g u g l i e l m o . l a n z a n i @ ~ f i s i . p o l i m i . i t . ~}$ ties of thin films of MEH-PPV, doped with $\mathrm{C}_{61}$-butyric acid methyl ester (PCBM).

In addition, we studied the photocurrent (PC) action spectrum of MEH-PPV sandwiched between a usual indium tin oxide (ITO) layer and cell culturing media, based on minimum essential medium (MEM) or physiological buffer solution, working as cathodes. We demonstrate that these unconventional, hybrid solid-liquid devices work as photodiodes, in photovoltaic conditions. Using liquid as a cathode allows us to circumvent the problem of metal cathode degradation mentioned above.

Potential changes in the optical and photophysical properties of MEH-PPV:PCBM thin films, caused by the prolonged contact with non deionized water, were studied by means of absorption and photoluminescence (PL) measurements, and by pump-probe spectroscopy (P\&P). The laser system used for the latter has been described elsewhere. ${ }^{8}$ $\mathrm{P} \& \mathrm{P}$ measures the differential change in transmission on subpicosecond time scale, identifying formation and recombination mechanisms of neutral and charged states in the polymer. One can calculate the $\Delta T / T$ spectrum at the specific probe delay $\tau$ as $\Delta T / T(\lambda, \tau)=\left[T_{\text {on }}(\lambda, \tau)-T_{\text {off }}(\lambda)\right] / T_{\text {off }}(\lambda)$, where $T_{\text {on(off) }}$ is the transmission with (without) pump.

The experimental setup for PC action spectra measurement has been previously described. ${ }^{5}$ All measurements are performed at room temperature and without applying bias.

Chlorobenzene solutions of MEH-PPV:PCBM (1:1 concentration, in weight) were deposited onto two glass substrates by spin-coating, using identical procedures. When not under testing, both samples were kept in the dark, one in vacuum, the other completely immersed in a pool of nondeionized water, for more than twenty hours.

The absorption and PL spectra of the "dry" and the "wet" samples are compared in Fig. 1, together with the chemical structure of the polymers. One can observe that after 1 day-immersion, there are no substantial differences in either the shape or the position of the absorption spectra. The PL spectra are also very similar, and no emission quenching is detected: This excludes the formation of nonradiative decay centers during immersion. The PL spectrum of the wet sample exhibits a more pronounced peak at $2 \mathrm{eV}$; this could be ascribed ${ }^{9}$ to a partial reorganization of the polymer chains due to a slight permeation of water into the organic film. This 


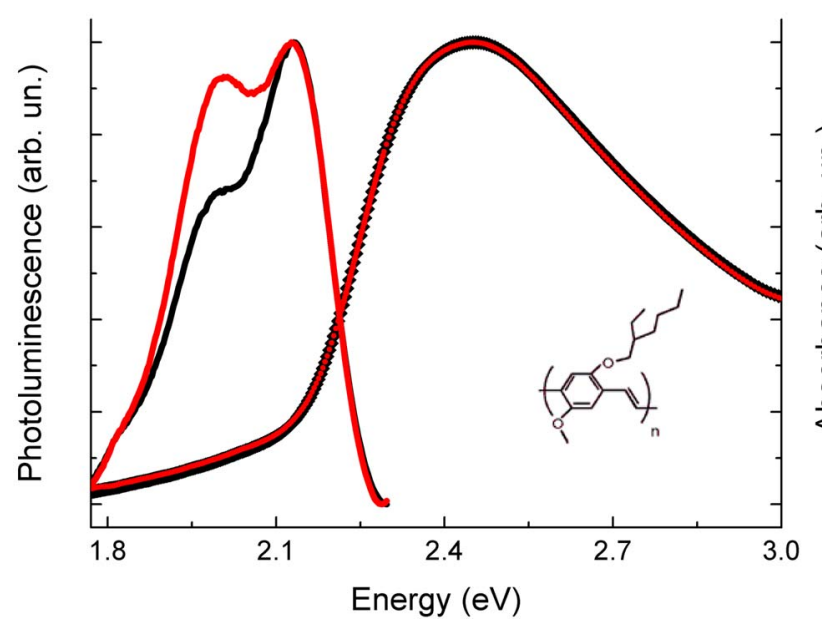

FIG. 1. (Color online) Absorption and PL spectra of MEH-PPV films, immediately after deposition (black lines) and after $24 \mathrm{~h}$ immersion in nondeionized water (red lines). Only the PL spectra show a difference in the relative intensity of the secondary peak at $2 \mathrm{eV}$, which is enhanced for the wet sample.

feature deserves further investigation, however, it is not relevant to the goal of this study.

Figure 2 shows experimental results obtained from $\mathrm{P} \& \mathrm{P}$ measurements in MEH-PPV:PCBM thin films. Transient absorption spectra at fixed probe delays (150 fs, $1 \mathrm{ps}$, and $380 \mathrm{ps}$ ) and temporal dynamics at two selected probe energies $(1.8$ and $2.7 \mathrm{eV})$ are reported. The positive signal at probe energies greater than $2.2 \mathrm{eV}$ is assigned ${ }^{10}$ to photobleaching $(\mathrm{PB})$ of the optical absorption, due to ground state population depletion; the photoinduced absorption (PA) band at lower energies is assigned to intrachain polarons, i.e., charged excited states. Photogeneration of the latter is sensitive to contamination that easily induces donor/acceptor impurities. From the spectral evolution, it is possible to note that the PA band is not instantaneously formed. Nevertheless, after $1 \mathrm{ps}$, the temporal dynamics at 1.8 and $2.7 \mathrm{eV}$ show the same behavior with similar decay times.

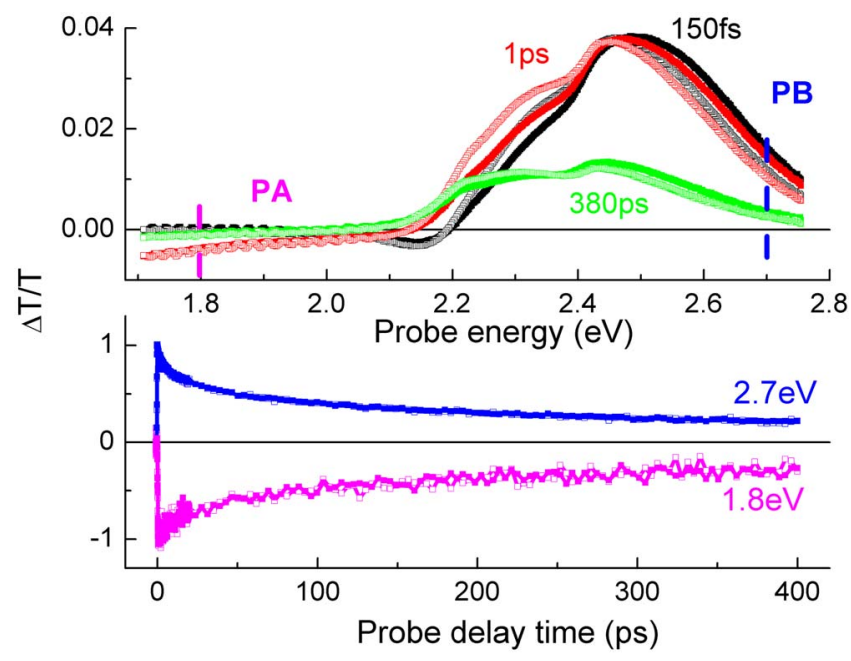

FIG. 2. (Color online) $\mathrm{P}$ and $\mathrm{P}$ spectra (upper) and dynamics (lower) of MEH-PPV:PCBM blend. Positive and negative band are assigned, respectively, to PB and PA of intrachain polarons. Transient decays (at 1.8 and 2.7 $\mathrm{eV}$ probe energies) and spectra at different probe delays (150 fs, $1 \mathrm{ps}$, and 380 ps after photoexcitation) of the dry (filled symbols) and the wet film (open symbols) do not present any relevant difference.

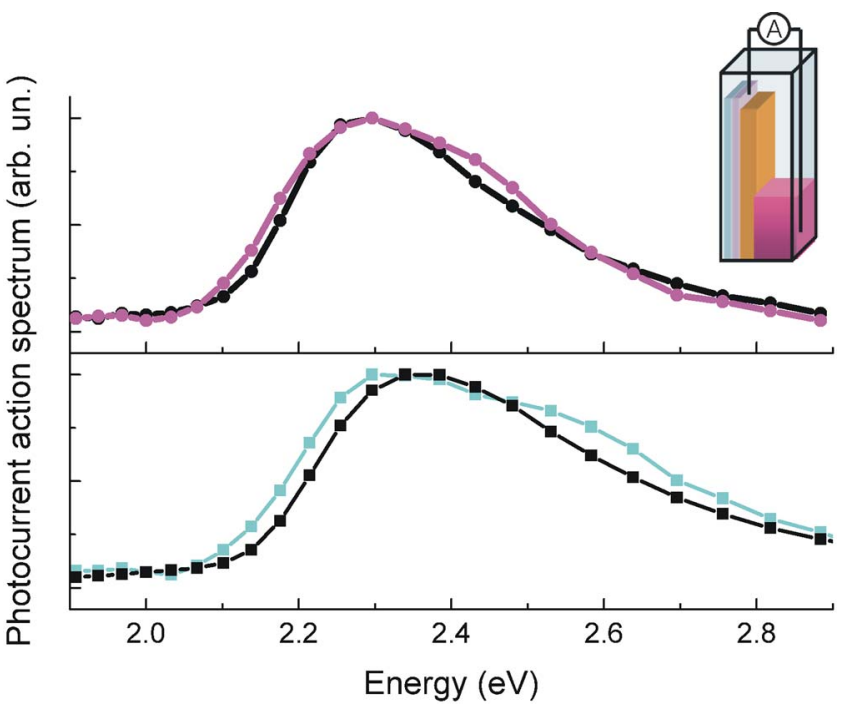

FIG. 3. (Color online) Normalized PC action spectra of usual sandwich photodiodes (ITO/MEH-PPV/Al, upper panel, circles black line, and ITO/ MEH-PPV:PCBM/Al, lower panel, squares black line), compared with those measured using a liquid cathode, MEM (upper, circles magenta line) and $\mathrm{KRH}$ (lower, squares cyan line). The inset reports a sketch of the hybrid photodiode: the polymer film is deposited onto ITO-coated substrates, properly etched; the cathode is a bath of MEM or KRH.

Experimental data show that the transient behavior for the water-immersed film strictly replicates the dry film; neither the intensity nor the lineshape of the P\&P signal seems to be affected by the prolonged contact with water. The formation and subsequent evolution of polaron states, which form the basis for optoelectronic device function, seem not to be compromised.

These results represent a clear indication that direct contact with water and oxygen has no relevant consequences on the photophysical characteristics of the MEH-PPV:C60 film. Analogous conclusions were reached in the case of the widely used polythiophene rr-P3HT (not shown here).

As a further step toward the realization of a direct interface between a thin film polymer device and a neural culture, we sterilized chlorobenzene MEH-PPV solutions using thermal sterilization and filtration (0.22 $\mu \mathrm{m}$ filters). The sterilized polymer was then deposited onto glass substrates covered with ITO (which acts as anode) by spin-coating. MEM with Earle's Salts without L-glutamine (purchased from Invitrogen) and a salt solution, namely a Krebs-Ringer's solution (KRH) buffered with (4-(2-hydroxyethyl)-1piperazineethanesulfonic acid (HEPES) were then used as cathodes, instead of commonly adopted metallic cathodes, thus producing a hybrid solid-liquid device. To avoid contact between the electrodes, the edges of the ITO-coated glass were etched and protected with polyetrafluoroethylene (PTFE). To extract the PC signal, a gold wire was immersed in the solution (Fig. 3, inset). As a reference, standard sandwich-structure photodiodes, both with and without PCBM, were produced by thermal evaporation of an aluminum cathode (thickness $\sim 120 \mathrm{~nm}$ ).

Figure 3 (upper) compares the normalized PC action spectra of pristine MEH-PPV using both aluminum and MEM solution as cathode; in the lower part of the figure, similar results are reported for MEH-PPV:PCBM devices, this time with metallic and KRH cathodes. Since the ionic content of KRH is much lower than that of MEM, it was 
necessary to dope the MEH-PPV with PCBM to enhance the external quantum efficiency. In both the pristine and the blend devices, the spectral shape of the PC action spectrum does not significantly depend on the cathode material. Typical peak values of the extracted PC through the liquid cathodes, in photovoltaic regime, were in the order of $10^{2} \mathrm{pA}$ up to nA, with dark current values in the order of $\sim 0.1 \mathrm{pA}$. These values are comparable to those obtained using conventional photodiodes, demonstrating the feasibility of our approach and the good properties of the liquid cathodes. The action spectra of hybrid photodiodes show only a slight broadening respect to the conventional photodiodes. We postulate that, as for the PL spectra, a certain amount of biological liquid components are incorporated into the polymer film, slightly changing the polymer chain configuration and possibly also affecting interchain interactions and charge carrier transport. Nevertheless, given that differences between solid and hybrid device characteristics are negligible, we deduce that the charge photogeneration mechanism is the same in both, namely singlet dissociation near the electrode collecting the slow carriers (cathode). ${ }^{11}$

The main difference between hybrid and conventional photodiodes is related to interface phenomena between the polymer film and the cathode. In the hybrid cell, the ionic transport inside the liquid media plays a key role, and the conductance type changes from (mainly) electronic to ionic at the semiconductor-electrolyte interface, ${ }^{12}$ while in the solid-state cell, the mechanism is that of a standard Schottkybarrier photodiode. Unfortunately, the electronic and ionic components of the current (and the ion entrance/exit through the polymer surface) are difficult to disentangle in most experiments, ${ }^{13}$ and particularly in the present case, where the whole surface of the polymer film is exposed to the liquid.

The interfaces between inorganic semiconductors and electrolytes have been studied in the past, taking into account electron transfer reactions. ${ }^{14}$ However, a detailed model of the mechanisms occurring at the organic, thin film polymer/ liquid electrolyte interface is still lacking, and the biological complexity of the media used in this work does not allow a rigorous physical description. In order to fully understand what happens at the polymer/liquid inferface, it will be necessary, in future investigations, to carefully control the physical-chemical properties (e.g., $p \mathrm{H}$, conductivity, ion content, etc.) of the liquid cathodes.
In conclusion, we demonstrate that polymer photodiodes are suitable for interfacing with the biological environment, that they remain unaltered during the procedures required for cell network growth and suggest a device configuration which eliminates the problem of the metallic cathode. Our results are important in the study of interfaces between living tissues and conducting polymers, more and more frequently used as protective layers in artificial prosthesis, and they also may open the way to the application of conducting polymers as active materials in neural implants.

${ }^{1}$ Semiconducting Polymers: Chemistry, Physics and Engineering, 2nd ed., edited by G. Hadziioannou and G. G. Malliaras (Wiley, Weinheim, 2007), Chap. 10, pp. 421-454.

${ }^{2}$ J. T. Mabeck and G. G. Malliaras, Anal. Bioanal. Chem. 384, 343 (2006); A. Loi, I. Manunza, and A. Bonfiglio, Appl. Phys. Lett. 86, 103512 (2005); P. Pistor, V. Chu, D. M. Prazeres, and J. P. Conde, Sens. Actuators B 123, 153 (2007); B. R. Panda and A. Chattopadhyay, J. Colloid Interface Sci. 316, 962 (2007).

${ }^{3}$ S. M. Richardson-Burns, J. L. Hendricks, B. Foster, L. K. Povlich, D. Kim, and D. C. Martin, Biomaterials 28, 1539 (2007); A. S. Widge, M. Jeffries-El, X. Cui, C. F. Lagenaur, and Y. Matsuoka, Biosens. Bioelectron. 22, 1723 (2007).

${ }^{4}$ J. Isaksson, P. Kjäll, D. Nilsson, N. D. Robinson, M. Berggren, and A. Richter-Dahlfors, Nature Mater. 6, 673 (2007); D. Feili, M. Schuettler, T. Doerge, S. Kammer, and T. Stieglitz, Sens. Actuators, A 120, 101 (2005); E. Bystrenova, M. Jelitai, I. Tonazzini, A. N. Lazar, M. Huth, P. Stoliar, C. Dionigi, M. G. Cacace, B. Nickel, E. Madarasz, and F. Biscarini, Adv. Funct. Mater. 18, 1751 (2008).

${ }^{5}$ M. R. Antognazza, U. Scherf, P. Monti, and G. Lanzani, Appl. Phys. Lett. 90, 163509 (2007).

${ }^{6}$ L. Lüer, H. J. Egelhaaf, D. Oelkrug, G. Cerullo, G. Lanzani, B. H. Huisman, and D. de Leeuw, Org. Electron. 5, 83 (2004).

${ }^{7}$ T. Someya, A. Dodabalapur, A. Gelperin, H. E. Katz, and Z. Bao, Langmuir 18, 5299 (2002); B. Huisman, J. J. P. Valeton, W. Nijssen, J. Lub, and W. ten Hoeve, Adv. Mater. (Weinheim, Ger.) 15, 2002 (2003); J. Ficker, A. Ullman, W. Fix, H. Rost, and W. Clemens, J. Appl. Phys. 94, 2638 (2003).

${ }^{8}$ D. Polli, L. Lüer, and G. Cerullo, Rev. Sci. Instrum. 78, 103108 (2007).

${ }^{9}$ S. R. Amrutha and M. Jayakannan, J. Phys. Chem. B 112, 1119 (2008).

${ }^{10}$ T. Drori, C. Sheng, A. Ndobe, S. Singh, J. Holt, and Z. Vardeny, Phys. Rev. Lett. 101, 037401 (2008).

${ }^{11}$ M. G. Harrison, J. Grüner, and G. C. W. Spencer, Phys. Rev. B 55, 7831 (1997); M. R. Antognazza, D. Musitelli, S. Perissinotto, and G. Lanzani (unpublished).

${ }^{12} \mathrm{H}$. Gerischer, in Physical Chemistry: An Advanced Treatise, edited by $\mathrm{H}$. Eyring (Academic, New York, 1970), Vol. IXA, pp. 463-542.

${ }^{13}$ X. Wang, B. Shapiro, and E. Smela, Adv. Mater. 16, 1605 (2004).

${ }^{14}$ H. Gerischer, Surf. Sci. 18, 97 (1969); R. Dogonadze, Prog. Surf. Sci. 6, 1 (1975) 